\title{
Fatigue assessment of a railway bridge detail using dynamic analysis and probabilistic fracture mechanics
}

\author{
B.M. Imam \& G. Kaliyaperumal \\ Faculty of Engineering and Physical Sciences, University of Surrey, Guildford, GU2 7XH, UK
}

\begin{abstract}
This paper presents a generic methodology for the use of PFM within the context of bridge loading for the fatigue design and assessment of steel railway bridges and provides detailed guidance on how to use the proposed methodology in order to carry out a PFM-based fatigue assessment. The problem is set in a probabilistic context to take into account material, loading as well as modeling uncertainties. Guidance is given on how to calibrate a constant amplitude PFM analysis against an S-N curve. Finally, as a case study, a cracked welded bridge detail is considered and its time-dependent fatigue reliability is established
\end{abstract}

\section{INTRODUCTION}

The use of probabilistic fracture mechanics (PFM) has been widespread in the offshore industry (Kirkemo 1998, Madsen et al. 1987, Shetty \& Baker $1990 \mathrm{a}, \mathrm{b}, \mathrm{c})$ unlike the bridge industry which has almost exclusively relied on the S-N approach and Miner's rule (Miner 1945). In the last two decades or so, there have been studies on the application on PFM in bridge structures (Chung et al. 2006, Cremona 1996, Kwon \& Frangopol 2011, Lukic \& Cremona 2001, Righiniotis 2004, Righiniotis \& Chryssanthopoulos 2004, Zhao \& Haldar 1996) but the topic still remains rather academic.

Use of standardised S-N curves can make it difficult to obtain the correct bridge condition assessment and the most appropriate maintenance strategy, especially in the case where fatigue cracks have been detected on a bridge. In many cases, crack detection points to immediate repair which may often be unnecessary. The Fracture Mechanics (FM)based approach is capable of capturing the propagation behaviour of fatigue cracks and quantifying their effect on fatigue life. Furthermore, the allimportant issue of inspections and repairs can be addressed through PFM which is also able to assist towards deciding on the most cost-effective maintenance strategies for steel bridges.

The time-dependent fatigue failure probability estimates are notoriously sensitive to crack and crack growth modelling, initial crack size, fatigue crack initiation life and other modelling uncertainties. In bridges, one such source of modelling uncertainty is typically expressed as the ratio $B_{s t}$ of the actual nominal stresses to the ones obtained through structural analysis modeling. In fatigue assessment, an accu- rate estimation of the resulting stresses is essential since they significantly affect fatigue life. Furthermore, the bridge engineer does not have in his/her disposal detailed guidance on the challenging application of PFM techniques and is faced with choosing appropriate models from a wide pool of published information, in many cases not readily available.

The objective of this paper is to present a generic methodology for the use of PFM within the context of bridge loading for the fatigue design and assessment of steel railway bridges and to provide detailed guidance on how to use the proposed methodology in order to carry out a PFM-based fatigue assessment. The problem is set in a probabilistic context to take into account material, loading as well as modelling uncertainties. Guidance is given on how to calibrate a constant amplitude PFM analysis against an S-N curve. Finally, as a case study, a cracked welded bridge detail is considered. Dynamic finite element (FE) analysis of the bridge is carried out and the reliability of the resulting stresses is established through comparison with field measurements to account for modeling uncertainties. By using the fatigue load spectra developed from typical railway traffic, fatigue life estimates are obtained via the PFM methodology.

\section{PROBABILISTIC FRACTURE MECHANICS}

\subsection{General considerations and failure criteria}

The stress field ahead of the crack tip, which is formed due to static loading, is described by the Stress Intensity Factor (SIF) $K_{a}$, which is specified 
by Linear Elastic Fracture Mechanics (LEFM) as (Anderson 2005)

$$
K_{a}=S Y_{a} \sqrt{\pi a}
$$

where $S$ is the externally applied stress, $a$ is the crack size and $Y_{a}$ is the Stress Magnification Factor (SMF), which depends on the geometry of the crack and the type of loading. For a given geometry and loading, $Y_{a}$ can be obtained from various sources (Murakami 1987, Tada et al. 2000). For an ideally brittle material, failure of a cracked component can occur when (Anderson 2005)

$$
K_{a}=K_{\text {mat }}
$$

where $K_{m a t}$ is the fracture toughness of the material. Fracture toughness generally increases with the increase in temperature and decreases with an increase in material thickness or strain rate (Cui 2002). A more exact way that takes into account possible ductile behaviour is by using the Failure Assessment Diagrams (FAD) available in BS 7910 (2005). When considering the FAD, the interaction between brittle and ductile behaviour is taken into account as well as the influence of different types of loading (external-primary and secondary - for example residual stresses). The use of a failure criterion, for given material properties ( $K_{m a t}$, yield stress $S_{y}$ and ultimate tensile stress $S_{u t s}$ ), can lead to the calculation of the maximum size of the crack $a_{f a i l}$ at failure.

According to the FAD failure criterion suggested in BS 7910 (2005), failure occurs when, for given loading and material properties, the crack size corresponds to a point that lies on or outside the failure envelope, which is defined as

$$
\begin{array}{ll}
K_{r}=\left(1-0.14 L_{r}^{2}\right)\left(0.3+0.7 e^{-0.65 L_{r}^{6}}\right) & L_{r} \leq L_{r, \max } \\
K_{r}=0 & L_{r}>L_{r, \max }
\end{array}
$$

The parameters in Equations 3 and 4 are defined as follows (BS 7910 2005)

$$
\begin{aligned}
& K_{r}=\frac{K}{K_{\text {mat }}}+\rho \\
& L_{r}=\frac{S_{r e f}}{S_{y}}
\end{aligned}
$$

where $\rho$, given in Annex R of BS 7910 (2005), represents the interaction between the external loading and the internal residual stresses and $K$ is given as

$$
K=K_{a}+K_{\text {res }}
$$

Expressions for residual stresses are given in BS 7910 (2005).
$S_{\text {ref }}$ in Equation (6) takes into account creep and plastic collapse and for normal bending restraint is given as

$S_{\text {ref }}=\frac{2 P_{b}}{3\left(1-a^{\prime \prime}\right)^{2}}$

where $P_{b}=S_{r}$ for constant amplitude loading and $P_{b}$ $=S_{\max }$ for variable amplitude loading since zerotension loading conditions were assumed. $S_{r}$ is the applied stress range. $a^{\prime \prime}$ depends on the geometry and is given in BS 7910. $L_{r, \max }$ in Equations 3 and 4 is given as (BS 7910 2005)

$$
L_{r \cdot \max }=\min \left[1.2 S_{y}, 0.5\left(S_{y}+S_{u t s}\right)\right] S_{y}{ }^{-1}
$$

In addition to the above criteria, an engineer must also ensure that the geometric constraints of the problem are also satisfied at each time. Many researchers agree that geometric (or serviceability) failure occurs when the crack length becomes equal to the thickness of the plate (Kirkemo 1998, Zhao et al. 1994), while others set this limit at half of the plate thickness (Cremona 1996).

In high cycle fatigue, which is predominant in steel bridges and where LEFM conditions are prevalent, the general crack growth relationship, also known as Paris law, is given as (Paris \& Erdogan 1963)

$$
\frac{d a}{d N}=C\left(\Delta K_{a}\right)^{m}
$$

where $C, m$ are material constants, $N$ is the number of the applied stress cycles and

$\Delta K_{a}=S_{r} Y_{a} \sqrt{\pi a}$

$C$ depends on the stress ratio $R$, which is higher for welded structures due to the effect of residual stresses and the environment (Schijve 2001, Suresh 1991). Equation 10 has been experimentally shown to be valid for different metals and steels of different yield strengths. Experiments have shown that a crack growth curve may possess a lower cut-off. Below this point, corresponding to a value $\Delta K_{t h r}$, Equation 10 no longer applies and $d a / d N=0$. The threshold stress intensity range $\Delta K_{t h r}$ is a material property and depends on the environmental conditions (Suresh 1991).

Equations 1, 10 and 11 can only treat cracks with one degree of freedom, for example through thickness cracks. However, in most cases, cracks propagate in two directions (depth and width) thus requiring a second degree of freedom for their description. A crack of this type, which has typically an elliptical/semi-elliptical shape, apart from Equation 10, requires a second similar differential equation for description of its growth. Accordingly, modifications that have been proposed for the $Y_{a}$ factors take into account both the shape of the crack (Newman \& 
Raju 1979) and the weld toe geometry (Hobbacher 1993).

\subsection{Fatigue life using the FM approach}

Under constant amplitude loading and by assuming a one-degree-of-freedom crack, solution of Equation 10 leads to

$$
N_{f}=\frac{1}{C S_{r}^{m}} \int_{a_{i n}}^{a_{f}} \frac{d a}{\left(Y_{a} \sqrt{\pi a}\right)^{m}}+N_{i n}
$$

where $N_{f}$ is the total number of applied stress cycles required to propagate the crack to a size $a_{f}$ and $N_{i n}$ is the number of cycles required to form an initial crack of size $a_{i n} . N_{\text {in }}$ is also known as the crack initiation period, while the integral corresponds to the crack propagation period. Having obtained $a_{\text {fail }}$ from Equation 2 or through a FAD, $a_{f}$ can be set equal to $a_{\text {fail }}$.

For the case of variable amplitude loading, Equation 12 may be written as (Cremona 1996, Kirkemo 1988, Lukic \& Cremona 2001)

$$
\frac{1}{C} \int_{a_{i n}}^{a_{f}} \frac{d a}{\left(Y_{a} \sqrt{\pi a}\right)^{m}}=\sum_{i=N_{i}}^{N_{f}} S_{r, i}^{m}
$$

Since the stress ranges $S_{r, i}^{m}$ represent the loading and are therefore random, the expected value of the right hand side of Equation 13 can be considered, leading to (Cremona 1996, Kirkemo 1988, Lukic \& Cremona 2001)

$$
\frac{1}{C} \int_{a_{i n}}^{a_{f}} \frac{d a}{\left(Y_{a} \sqrt{\pi a}\right)^{m}}=\left(N_{f}-N_{i}\right) E\left[S_{r}^{m}\right]
$$

The limit state function for the PFM-based fatigue assessment may be written as

$$
g(X, t)=\frac{1}{C} \int_{a_{i n}}^{a_{f}} \frac{d a}{\left(Y_{a} \sqrt{\pi a}\right)^{m}}-\left[N_{f}(t)-N_{i}(t)\right] E\left[S_{r}{ }^{m}\right]
$$

In Equation 15, the first terms represents the resistance and the second term the loading. Randomness in loading is captured through the expectation of $S_{r}^{m}$ as well as the annual number of applied cycles, which define the functional relationship between $N$ and $t$ (Righiniotis 2004).

\subsection{Methodology for predicting fatigue life}

In order to determine the fatigue life $N_{f}$, Equations 12 and 14, for constant and variable amplitude loading respectively, may be solved using numerical integration. Alternatively, Equation 3 may be solved using a finite difference scheme, a method which is suggested here. The steps are specified in terms of a fixed-cycle increment $\Delta N$ :

(a) Using the initial crack depth $a_{i n}$ and the other geometric parameters of the problem, the factor $Y_{a}$ can be calculated.

(b) Equation 1 is used to calculate $K_{a}$ which is then used to check failure of the detail. This can be done using a FAD or Equation 2. In the case of a specified serviceability criterion, for example the crack depth reaching a certain size, a similar check may be carried out in terms of the predefined failure crack size.

(c) Judicious selection of the step $\Delta N$ for the number of the applied stress cycles and the use of Equations 10 and 11 leads to the corresponding crack extension: $\Delta a=C \Delta K_{a}{ }^{m} \Delta N$

The number of elapsed cycles is increased by $\Delta N$, while the crack depth is increased by $\Delta a$, therefore:

$$
\begin{aligned}
& N_{i+1}=N_{i}+\Delta N \\
& a_{i+1}=a_{i}+\Delta a
\end{aligned}
$$

where $N_{i}$ and $a_{i}$ are equal to $N_{i n}$ and $a_{i n}$ for the first iteration.

The calculation returns to step (a) using the new crack size and this procedure continues until one (or both) of the second step's checks is (are) violated.

\section{$2.4 S$-N based FM model calibration}

In order to carry out a PFM-based analysis for variable amplitude loading, it is expedient to examine the behaviour of the model under constant amplitude loading and compare it with experimental results or the appropriate S-N curve. A calibration procedure needs to be carried out in order to verify the model assumptions. The models describing fatigue crack growth (distributions and their parameters for $C, m$, $K_{m a t}$, etc) are fairly well established. On the other hand, the initial crack size $a_{i n}$ is a highly uncertain variable and, according to Equations 12 and 14, is expected to have a significant influence on fatigue life estimates and associated fatigue reliability. This is the reason why, in many cases in the past, $a_{i n}$ has been used as the calibrating parameter to achieve good agreement between the life predictions given by the FM method with their code specified counterparts (Righiniotis \& Chryssanthopoulos 2003, 2004).

On the other hand, in other studies (Pedersen et al. 1992), the S-N calibration random parameter used was the crack initiation time $N_{\text {in }}$ in Equation 12. $N_{i n}$ was assumed to be given by (Pedersen et al. 1992)

$$
N_{\text {in }}=K_{1} S_{r}^{-m_{1}}
$$


where $K_{1}, m_{1}$ are material parameters. $m_{1}$ is deterministic while $K_{1}$ is lognormally distributed. Its mean value and standard deviation are obtained by calibrating the FM curves (mean and design) against the corresponding S-N curves of the code of practice adopted. Here, the use of BS 5400 (1980) is proposed since the Eurocode (EN1993-1-9 2005) does not include a mean curve. The steps followed when carrying out this calibration are:

(a) According to Equation 12, $N_{f}=N_{\text {prop }}+N_{i n}$, where $N_{\text {prop }}$ is the crack propagation time stage. Thus, for each stress range, the value of the mean FM curve is obtained from the value of the mean S-N curve. The outcome of this is assumed to be the mean value of the initiation time for this specific stress range. Using these values, the $\mathrm{E}\left[N_{i n}\right]$ (mean initiation time) versus $S_{r}$ graph can be plotted.

(b) A curve of the type of Equation 18 is fitted to this graph. $\mathrm{E}\left[K_{l}\right]$ and $m_{1}$ are taken equal to the parameters of the curve.

(c) $K_{1}$ is assumed to be lognormally distributed and various different values for its $\mathrm{CoV}$ are tested until the FM curves fit exactly on the corresponding S-N curves.

\section{CASE STUDY}

\subsection{Geometric description of bridge detail}

The bridge detail, which is considered here as a case study, forms part of the Söderstrom bridge in Sweden and was constructed in the 1950s. It consists of a main edge girder, which is $3000 \mathrm{~mm}$ deep and $600 \mathrm{~mm}$ wide. The girder has fillet-welded on its web a transverse stiffener. Figure 1 shows the configuration of the detail. The stiffeners are placed at equal spaces of $3370 \mathrm{~mm}$. The weld angle is assumed to be $45^{\circ}$. Figure 2 also shows pictures of the detail with the fatigue crack detected on the bridge following inspections. Such fatigue cracks were detected in most of the stiffener details on the bridge.

Table 1 presents the geometric parameters of the problem which are assumed to be deterministic. For $\mathrm{S}-\mathrm{N}$ calibration purposes, the stiffener is treated as a longitudinal attachment subjected to the out of plane bending stresses of the web. Thus, the detail may be classified as F2 according to BS 5400 (1980) and 56 based on EN1993-1-9 (2005). The plate is restrained in bending. As a result, the membrane stress $P_{m}$ may be neglected leaving the bending stress $P_{b}$ as the only primary external source of loading (BS7910 2005).

The crack is assumed to be half circular $(a / c=1)$ and is modelled as one-degree-of-freedom with the crack size growing in the depth of the girder web. Crack growth is assumed to be described by Equation 10 with no lower cut-off $\left(\Delta K_{t h r}=0\right)$.
The SMF for bending or tension ( $H$ factors equal to 1 in this case) can be derived by (Newman \& Raju 1979)

$Y_{a}=\frac{H_{A} M_{m A} M_{k m A}}{\sqrt{Q}}$

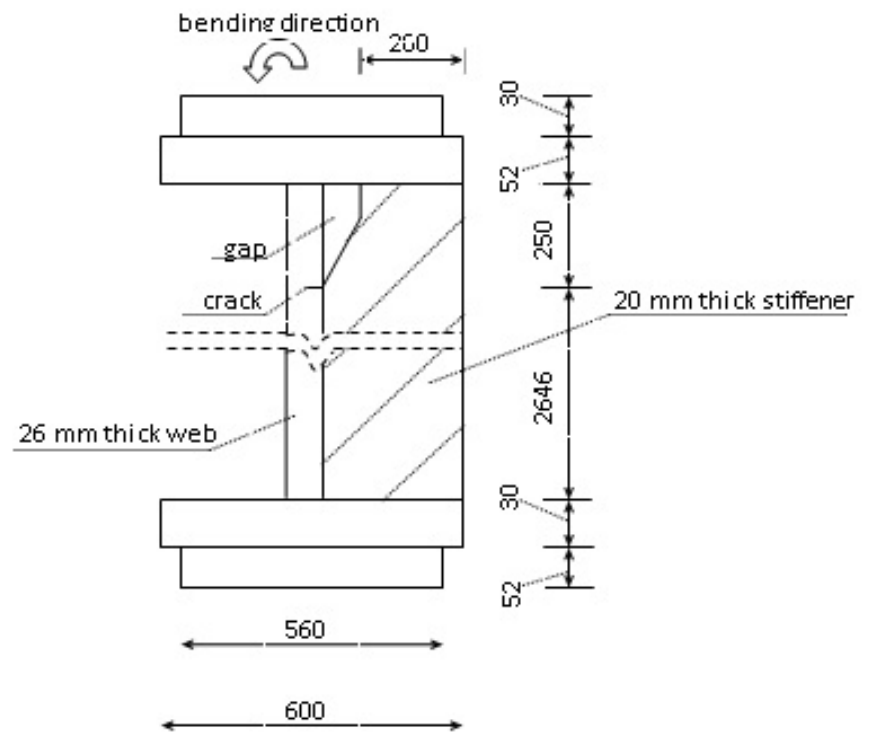

Figure 1. Configuration of bridge detail (all dimensions in $\mathrm{mm})$.
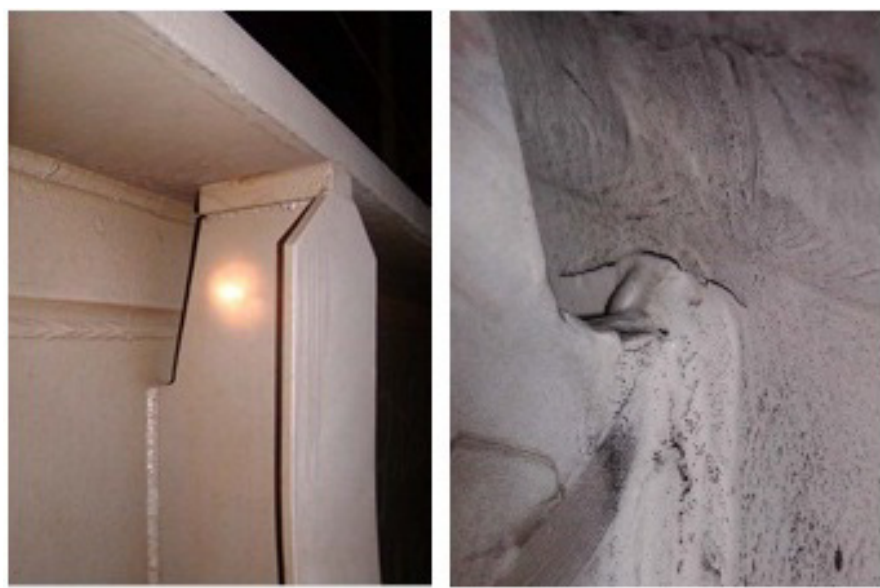

Figure 2. Actual bridge detail and fatigue crack observed.

Table 1. Geometric (deterministic) parameters of the problem.

\begin{tabular}{lcc}
\hline Parameter & Symbol & Value \\
\hline Plate thickness & $b$ & $26 \mathrm{~mm}$ \\
Attachment thickness & $t$ & $20 \mathrm{~mm}$ \\
Attachment length & $L_{\text {att }}$ & $2646 \mathrm{~mm}$ \\
Plate width & \multirow{2}{*}{${ }^{w}$} & $3370 \mathrm{~mm}$ \\
Weld angle & & $45^{\circ}$
\end{tabular}

where $H_{A}$ and $M_{m A}$ are the Newman-Raju (1979) plate solutions for point A, $Q$ is the shape correction factor (BS7910 2005, Newman \& Raju 1979) and $M_{k m A}$ is the welded geometry related SMF for the same point given as (Newman \& Raju 1979)

$M_{k m A}=u\left(\frac{a}{b}\right)^{w}$ 
where $u$ is given by (Hobbacher 1993)

$$
\begin{aligned}
u= & 0.9089-0.2357\left(\frac{t}{b}\right)+0.0249\left(\frac{L_{\text {att }}}{b}\right) \\
& -0.00038\left(\frac{L_{\text {att }}}{b}\right)^{2}+0.0186\left(\frac{w}{b}\right)-0.1414\left(\frac{\theta}{45^{\circ}}\right)
\end{aligned}
$$

and $w$ can be obtained from (Hobbacher 1993)

$$
\begin{aligned}
w= & -0.02285+0.0167\left(\frac{t}{b}\right)-0.3863\left(\frac{\theta}{45^{\circ}}\right) \\
& +0.123\left(\frac{\theta}{45^{\circ}}\right)^{2}
\end{aligned}
$$

It has to be mentioned that the parameter $u$ depends upon the ratios $w / b$ and $L_{a t t} / b$, which, for this configuration, were both outside of their range of validity. Both ratios are here set equal to a limiting value of 40 , which is the cut-off for these equations developed by Hobbacher (1993).

\subsection{Random variables}

The Paris parameter $C$ is assumed to be lognormally distributed with mean value of $5.86 \times 10^{-13}$ and $\mathrm{CoV}$ 0.6 (King et al. 1996). The Paris exponent $m$ is taken as deterministic and equal to 3 (Kirkemo 1988). The yield stress is assumed to be lognormally distributed with a mean value $350 \mathrm{MPa}$ and $\mathrm{CoV}$ of 0.07 (JCSS 2001). $S_{y}$ and $S_{u t s}$ were assumed to be fully correlated with $S_{u t s}=1.5 S_{y}$.

The initial crack depth was taken as exponentially distributed with mean $0.11 \mathrm{~mm}$ and $\mathrm{CoV}$ of 1.0 (Bokalrud \& Karlsen 1981). The crack initiation time was, for the first part of the analysis, taken as deterministic and equal to 0 due to the fact that $N_{i n}$ is generally negligible for welded details (Maddox 1991). However, in subsequent analyses it was used as the calibrating parameter for the F2 S-N curves provided in BS 5400 (1980). $N_{\text {in }}$ was for this purpose assumed to be of the form of Equation 18 (Pedersen et al. 1992). The entire procedure that was followed for the S-N calibration has been described in section 2.4 .

The fracture toughness was assumed to be Weibull distributed with a mean value of 2250 $\mathrm{N} / \mathrm{mm}^{3 / 2}$ and a CoV of 0.25 . Following the recommendations given in Burdekin \& Hamour (2000), the values that were chosen for the detail under consideration appear to be reasonable, bearing in mind that the web thickness is $26 \mathrm{~mm}$.

Residual stresses were also considered in the analysis. In general, the distribution of the actual residual stresses does not follow a linear or other simple pattern through the thickness of the plate and is random. However, as part of this analysis, the residual stresses were assumed to be constant (through the web thickness) and their randomness was only captured through the yield stress.

\section{RESULTS AND DISCUSSION}

\subsection{Dynamic analysis of the bridge}

Time history dynamic FE analysis of the bridge was carried out in order to obtain nominal stresses for the detail in question and produce a stress range spectrum for the estimation of the fatigue reliability of the detail. More information on these analyses can be found in Kaliyaperumal et al. (2011) where the reliability of the FE bridge model in predicting accurate stress histories has been established through comparison with field measurements. The comparisons were carried out in terms of $\mathrm{E}\left[S_{r}\right]$ since it is an important variable in terms of fatigue life estimation. Figures 3 and 4 show the comparisons between field measurements and dynamic FE analysis for different locations on the bridge (Kaliyaperumal et al. 2011) and for two test train velocities.

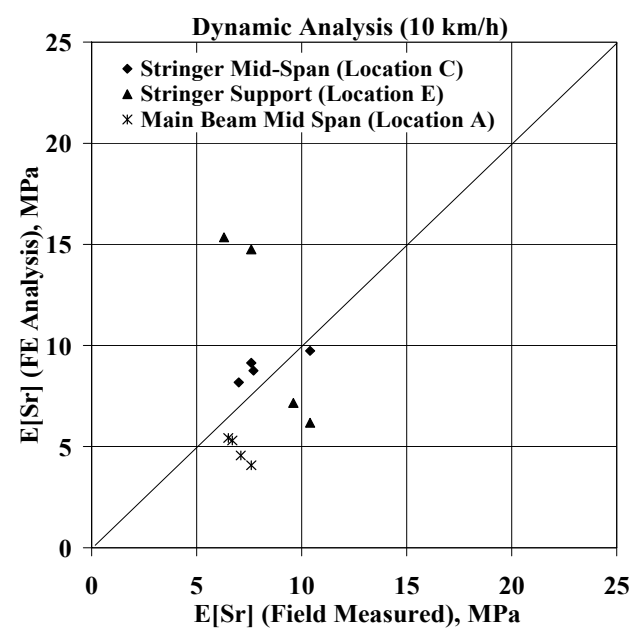

Figure 3. Comparison of $\mathrm{E}\left[S_{r}\right]$ between field measurements and $\mathrm{FE}$ dynamic analysis under train velocity of $10 \mathrm{~km} / \mathrm{h}$.

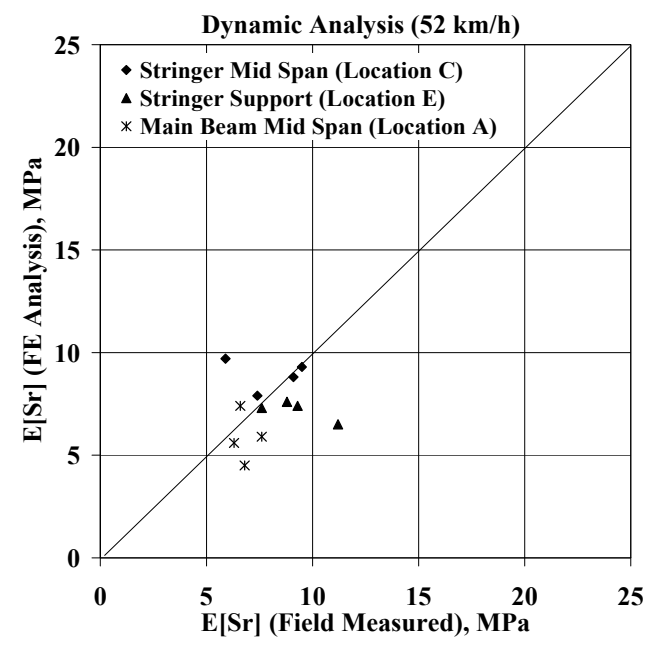

Figure 4. Comparison of $\mathrm{E}\left[S_{r}\right]$ between field measurements and FE dynamic analysis under train velocity of $52 \mathrm{~km} / \mathrm{h}$.

By using the results from Figures 3 and 4, an uncertainty factor has been developed to represent the modeling uncertainty $B_{s t}$ which represents the ratio of the actual nominal stress to the modeled nominal stress. By forming the measured (field) to modeled (FE) ratios for $\mathrm{E}\left[S_{r}\right]$, for all points on the bridge, a distribution for the $\mathrm{B}_{\text {st }}$ factor was developed and 
shown in Figure 5. The best fit to the histogram was found to be given by the lognormal distribution with mean and CoV values of 1.01 and 0.55 , respectively. The mean value of the uncertainty factor agrees well with quoted values in the literature where it is often assumed as 1.0 (Cremona 1996, Lukic \& Cremona 2001). However, its $\mathrm{CoV}$ is relatively high which means that it will contribute to the uncertainty in fatigue life estimates.

Figure 6 shows the details of trains which comprise the traffic running over the bridge and which were used for the FE analyses. The first train, consisting of axles $\mathrm{P} 1$ and $\mathrm{P} 2$ is a X60 commuter train with a frequency of 622 trains/day. The remaining two trains are the T11 and T12 freight trains suggested by EN 1991-2 (2003), both having a frequency of 16 trains/day.

Figure 7 shows the annual nominal stress range histogram obtained by running the three trains over the bridge. The expected value (mean) of the stress range $\mathrm{E}\left[S_{r}\right]$ was estimated as $11.2 \mathrm{MPa}$ with a $\mathrm{CoV}$ of 0.81 . The histogram shown in Figure 7 can also be used to estimate the value of $\mathrm{E}\left[S_{r}^{m}\right]$, which governs the fatigue life (see Equations 14 and 15).

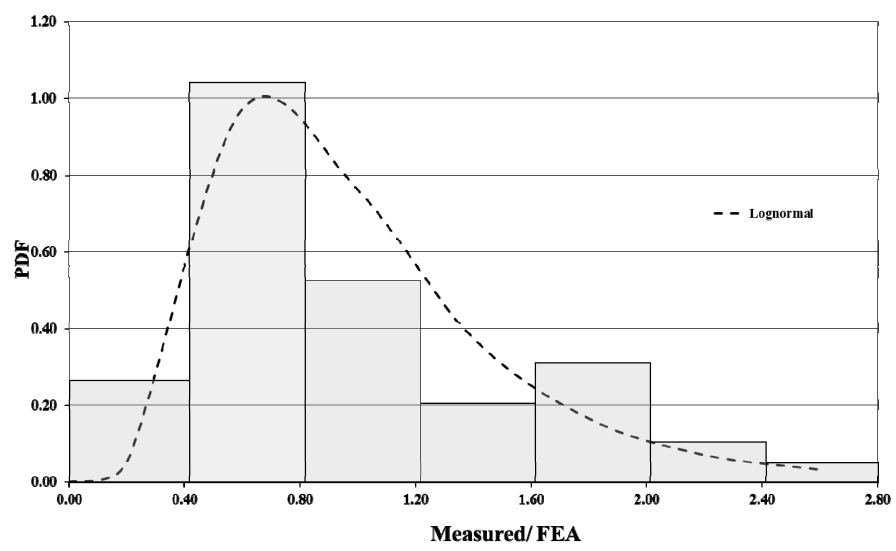

Figure 5. Model uncertainty factor $B_{s t}$ obtained from comparisons of field measurements with FE analysis.
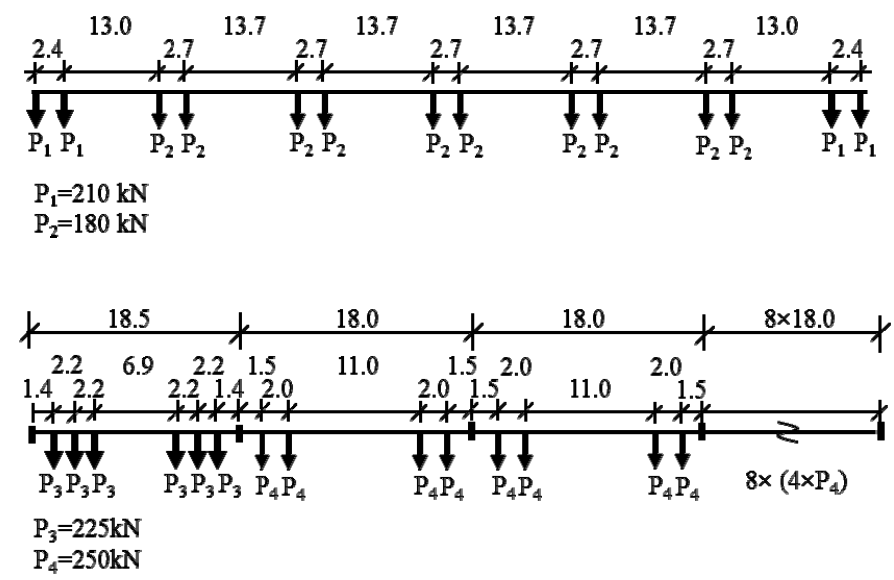

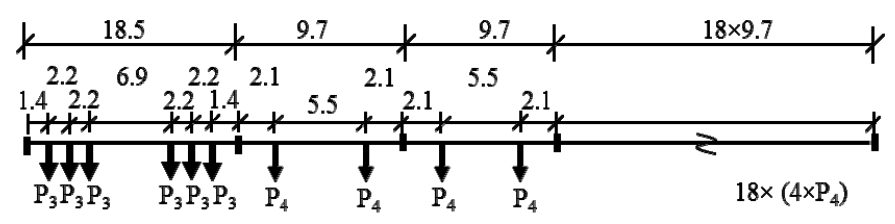

$\mathrm{P}_{3}=225 \mathrm{kN}$

$\mathrm{P}_{4}=250 \mathrm{kN}$

Figure 6. Details of train traffic on bridge.

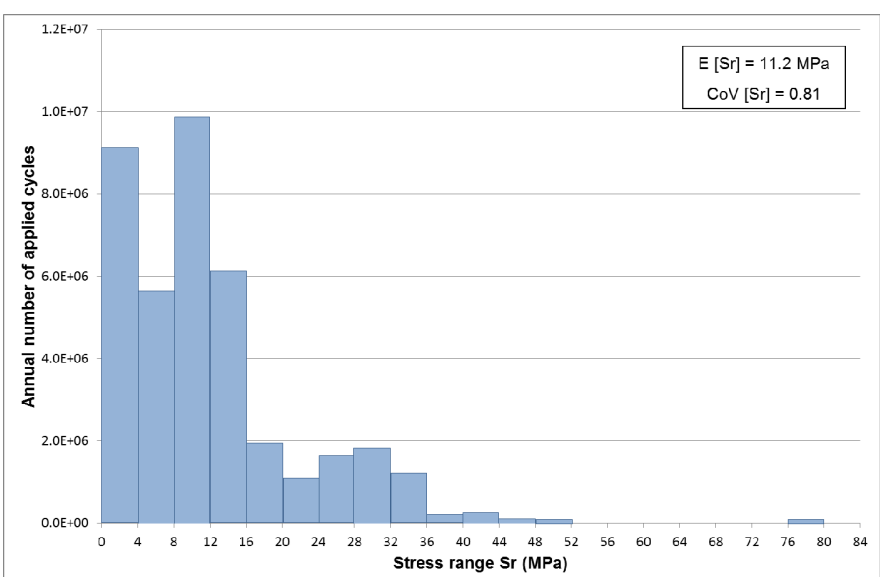

Figure 7. Annual nominal stress range histogram for bridge detail.

\subsection{S-N based FM model calibration}

For the purposes of the case study, Equation 10 was solved by using finite differences as described previously in section 2.3. The random variables of the problem were generated by using Monte Carlo simulation with $10^{5}$ samples. The crack was checked for failure at each increment through both the FAD as well as the condition of the crack penetrating the web.

The results of the first part of the analysis, where $N_{\text {in }}$ was assumed to be zero, are illustrated in Figure 8 , where the constant amplitude stress range is plotted on a logarithmic scale against the logarithm of the applied number of stress cycles at failure. In the same figure, the BS 5400 (1980) mean and 97.7\% (design) probability of survival lines for class F2 alongside with the Eurocode 95\% (design) probability of survival line for class 56 are presented. These lines are plotted together with the corresponding (mean, 95\% and $97.7 \%$ probabilities of survival) FM lines. Experimental data on longitudinal attachments taken from Maddox (1982) are also shown. The lines that were reproduced here from the codes of practice do not take into account any treatment of low stress cycles. The parallel characteristic of the lines in Figure 8 is the result of both the S-N approach and the FM approach using the same Paris parameter, namely, $m=3$. The results show a considerable discrepancy between the FM and the corresponding code specified lines. The very low fatigue lives obtained from FM may be attributed to the very large stress concentrations that are introduced through the factor 
$M_{k m A}$ for this specific bridge detail. This observation demonstrates that, unless unrealistically small initial crack sizes are used, a $N_{\text {in }}$ would need to be introduced in the analysis as a basis of calibration. However, the use of very small values of $a_{i n}$ is not recommended for two reasons. Firstly, because very short crack growth cannot be described by Equation 10 and secondly because calibration on $a_{i n}$ will, in general, affect inspection outcomes under bridge loading. Therefore, the use of $N_{i n}$ as a calibrating parameter appears to be particularly attractive. The results of the calibration based on $N_{i n}$ are shown in Figure 9. The match of the BS 5400 and the FM lines is almost perfect. The statistics of the random variable $K_{l}$ were found to be $\mathrm{E}\left[K_{l}\right]=3.16 \times 10^{11}$ and $\mathrm{CoV}\left[K_{l}\right]=0.5-0.55$, while $m_{1}$ was determined as equal to 2.846 .

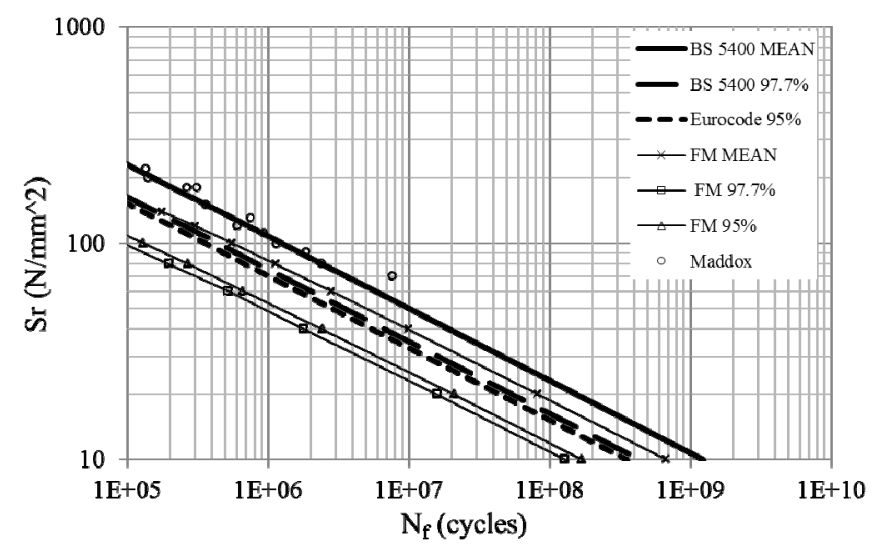

Figure 8. Comparison between the BS / Eurocode and FM lines.

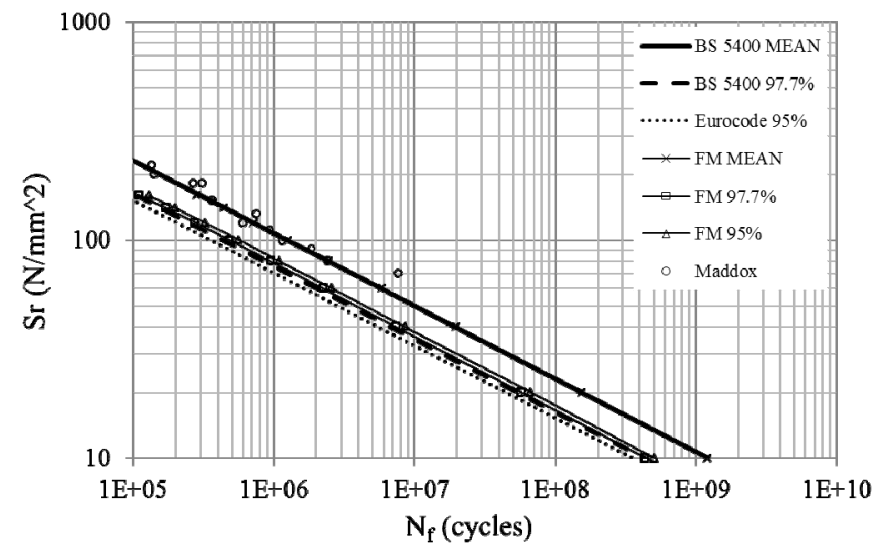

Figure 9. Calibration of the FM lines against the BS 5400 lines.

\subsection{Fatigue reliability}

Following the calibration of the FM curve with the $\mathrm{S}-\mathrm{N}$ curve for constant-amplitude loading using the initiation life $N_{i n}$ as the calibrating parameter, the variable-amplitude bridge loading was taken into account. By using the load spectrum developed for the detail (Figure 7), the probability of failure over time of the welded detail has been estimated through Equation 15 and is shown in Figure 10 labelled "basic". Furthermore, the effect of considering the cut-off limit of detail $56(23 \mathrm{MPa})$, suggested in EN1993-1-9 (2005) for neglecting nominal stress ranges using the $\mathrm{S}-\mathrm{N}$ method, has also been investigated as shown in Figure 10. It is evident from the results that the combination of the cut-off limit and the modeling uncertainty $B_{s t}$ has a small influence on the reliability of the particular bridge detail. This can be attributed to the fact that all stress ranges below the $23 \mathrm{MPa}$ cut-off limit may be unable to propagate the cracks by appreciable amounts and it is the larger stress ranges that give rise to significant crack growths.

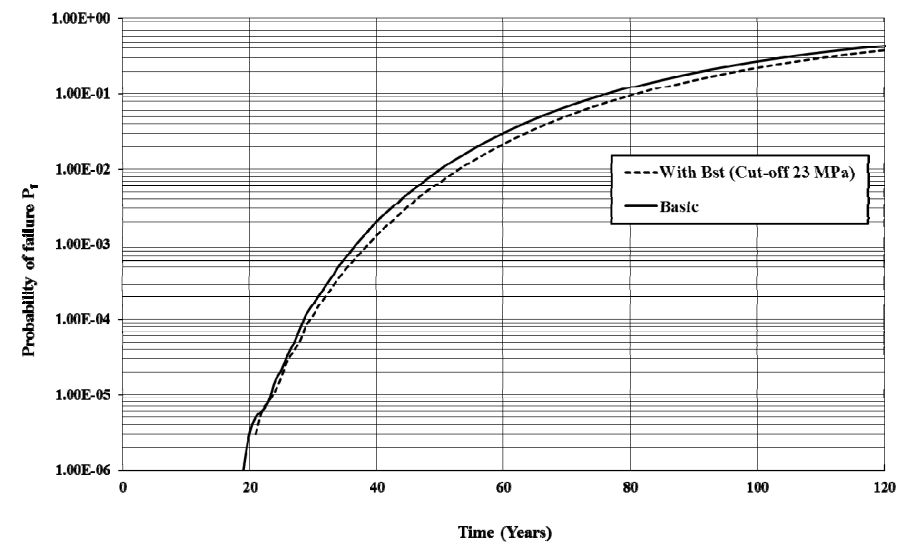

Figure 10. Probability of fatigue failure over time for case study bridge detail.

\section{CONCLUSIONS}

This paper has presented a robust PFM methodology for estimating the fatigue reliability of bridge details and its applicability has been demonstrated for a case study typical bridge detail under realistic bridge loading. PFM S-N curves were initially obtained for the bridge detail using Monte Carlo simulation. Through comparison with code-specified S-N curves, the results have shown that the use of values that were proposed in the literature for material properties and initial crack sizes can result in overly conservative fatigue life estimates. Given the inability of a Paris-type crack growth model to describe the growth of very short cracks as well as the influence of $a_{i n}$ selection on subsequent inspection results, it is here proposed that, within the context of a PFM analysis, the crack initiation time is used as an $\mathrm{S}-\mathrm{N}$ calibrating parameter. For this case study, calibration on this basis resulted in excellent agreement between the PFM and the code-specified S-N curves.

The developed generic PFM methodology can be easily adapted for application to any steel bridge detail and therefore, may be of benefit to bridge engineers. Through the application of PFM, which explicitly considers the behaviour of fatigue cracks, a more reliable condition assessment of a fatigue de- 
tail can be carried out. This may result in savings from unnecessary repair/replacement actions on cracked bridge details, thus prolonging the service life. Undoubtedly, the most appealing feature of the PFM methodology developed is its capability to account for the effect of inspection and repair actions on fatigue reliability.

\section{ACKNOWLEDGEMENTS}

This work was carried out with a financial grant from the Research Fund for Coal and Steel (RFCS) of the European Community, granted under the contract Nr. RFSR-CT-2008-00033. The authors sincerely acknowledge the division of structural design and bridges at the Royal Institute of Technology $(\mathrm{KTH})$ Sweden, structural engineering division at the Chalmers University of Technology Sweden and the Swedish Rail administration for providing the field measurements.

\section{REFERENCES}

Anderson, TL. 2005. Fracture mechanics: fundamentals and applications. London: Taylor \& Francis.

Bokalrud, T. \& Karlsen, A. 1981. Probabilistic fracture mechanics evaluation of fatigue failure from weld defects in butt weld joints. Im Conference on fitness for purpose validation of welded structures, Paper 28.

BS 5400: Part 10. 1980. Steel, concrete and composite bridges, Code of practice for fatigue. London: BSI.

BS 7910. 2005. Guide to methods for assessing the acceptability of flaws in metallic structures. London: BSI.

Burdekin, F.M. \& Hamour, W. 2000. Partial safety factors for SINTAP procedure. Offshore Technology Report, OTO 2000 020. London: HSE.

Chung, H.Y., Manuel, L. \& Frank, K.H. 2006. Optimal inspection scheduling of steel bridges using nondestructive testing techniques. Journal of Bridge Engineering (ASCE) 11(3): 305-319.

Cremona, C. 1996. Reliability updating of welded joints damaged by fatigue. International Journal of Fatigue 18(8): $567-575$.

Cui, W. 2002. A state-of-the-art review on fatigue life prediction methods for metal structures. Journal of Marine Science and Technolology 7(1): 43-56.

EN 1991-2. 2003. Eurocode 1: Actions on structures. Part 2 Traffic loads on bridges. London:BSI.

EN 1993-1-9. 2005. Eurocode 3: Design of steel structures Part 1.9: Fatigue. CEN.

Hobbacher, A. 1993. Stress intensity factors of welded joints. Engineering Fracture Mechanics 46(2):173- 182.

Joint Committee on Structural Safety (JCSS). 2001. The probabilistic model code. Internet Publication, http://www.jcss.ethz.ch.

Kaliyaperumal, G., Imam, B. \& Righiniotis, T. 2011. Advanced Dynamic Finite Element Analysis of a Skew Steel Railway Bridge. Engineering Structures 33(1): 181-190.

King, R.N., Stacey, A. \& Sharp, J.V. 1996. A review of fatigue crack growth rates for offshore steels in air and seawater environments. In Proceedings of the $15^{\text {th }}$ International Conference on Offshore Mechanical and Arctic Engineering, OMAE, 341-348.
Kirkemo, F. 1988. Applications of probabilistic fracture mechanics to offshore structures. Applied Mechanics Reviews 41(2): 61-84.

Kwon, K. \& Frangopol, D.M. 2011. Bridge fatigue assessment and management using reliability-based crack growth and probability of detection models. Probabilistic Engineering Mechanics 26: 471-480.

Lukić, M. \& Cremona, C. 2001. Probabilistic assessment of welded joints versus fatigue and fracture. Journal of Structural Engineering (ASCE) 127(2): 211-218.

Maddox, S. 1991. Fatigue strength of welded structures. Cambridge: Abington Publishers.

Maddox, S.J. 1982. Improving the fatigue lives of fillet welds by shot peening. In Proceedings of the IABSE Colloquium, Fatigue of Steel and Concrete Structure, 377-384, Lausanne.

Madsen, H.O., Skjong, R.K., Tallin, A.G. \& Kirkemo, A. 1987. Probabilistic fatigue crack growth analysis of offshore structures, with reliability updating through inspection. In Proceedings of SNAME Marine Structural Reliability Symposium, Arlington, Virginia, USA.

Miner, M.A. 1945. Cumulative damage in fatigue. Journal of Applied Mechanics (ASME) 12: 159-164.

Murakami, Y. 1987. Stress intensity factors handbook. Oxford: Pergamon Press.

Newman, J.C. \& Raju, I.S. 1979. Analyses of surface cracks in finite plates under tension or bending loads, NASA Technical Paper, No TP 1578.

Paris, P.C. \& Erdogan, F. 1963. A critical analysis of crack propagation laws. Journal of Basic Engineering (ASME) 85: 528-534, 1963.

Pedersen, C., Nielsen, J.A., Riber, J.P, Madsen, H.O \& Krenk, S. 1992. Reliability based inspection planning for the Tyra field. In Proceedings of the 11th OMAE Offshore Mechanics and Arctic Engineering Conference. ASME, 255-263.

Righiniotis, T.D. 2004. Influence of management actions on fatigue reliability of a welded joint. International Journal of Fatigue 26: 231-239.

Righiniotis, T.D. \& Chryssanthopoulos, M.K. 2004. Fatigue and fracture simulation of welded bridge details through a bi-linear crack growth law. Structural Safety 26: $141-158$.

Righiniotis, T.D. \& Chryssanthopoulos, M.K. 2003. Probabilistic fatigue analysis under constant amplitude loading. Journal of Constructional Steel Research 59(7): 867-886.

Schijve, J. 2001. Fatigue of structures and materials; Kluwer Academic Publishers.

Shetty, N.K. \& Baker, M.J. 1990. Fatigue reliability of tubular joints in offshore structures: Fatigue loading. In Proceedings of the $9_{\text {th }}$ Offshore Mechanics and Arctic Engineering Conference. ASME, 215-222.

Shetty, N.K. \& Baker, M.J. 1990. Fatigue reliability of tubular joints in offshore structures: Crack propagation model. In Proceedings of the $9_{\text {th }}$ Offshore Mechanics and Arctic Engineering Conference. ASME, 223-230.

Shetty, N.K. \& Baker, M.J. 1990. Fatigue reliability of tubular joints in offshore structures: Reliability analysis. In Proceedings of the 9 $9_{\text {th }}$ Offshore Mechanics and Arctic Engineering Conference. ASME, 231-239.

Suresh, S. 1991. Fatigue of materials. Cambridge: Cambridge University Press.

Tada, H., Paris, P.C. \& Irwin, G.R. 200. The stress analysis of cracks handbook. New York: ASME.

Zhao, Z., Haldar, A. \& Breen, F.L. 1994. Fatigue reliability evaluation of steel bridges. Journal of Structural.Engineering (ASCE) 120(5): 1624-1642.

Zhao, Z. \& Haldar, A. 1996. Bridge fatigue damage evaluation and updating using non-destructive inspections. Engineering Fracture Mechanics 53(5): 775-788. 